\title{
Mechanisms of U-Shaped Association between Alcohol Intake and the Risk of Sudden Cardiac Death
}

\author{
Hrayr S Karagueuzian ${ }^{1 *}$, and Levon Demirdjian ${ }^{2}$ \\ ${ }^{1}$ Director Translational Arrhythmia Research Section, Cardiovascular Research Laboratories, David Geffen School of Medicine at University of \\ California, USA \\ ${ }^{2}$ Center for Computational and Genomic Medicine, Children's Hospital of Philadelphia, USA
}

Received: 01 Mar, 2019 | Accepted: 22 Apr, 2019 | Published: 29 Apr, 2019

*Corresponding author: Karagueuzian HS, Director Translational Arrhythmia Research Section, Cardiovascular Research Laboratories, David Geffen School of Medicine at University of California, Los Angeles, USA, E-mail: hkaragueuzian@mednet.ucla.edu

Citation: Karagueuzian HS, Demirdjian L (2019) Mechanisms of U-Shaped Association between Alcohol Intake and the Risk of Sudden Cardiac Death. J Hear Health 5(1): dx.doi.org/10.16966/2379-769X.149

Copyright: (c) 2019 Karagueuzian HS, et al. This is an open-access article distributed under the terms of the Creative Commons Attribution License, which permits unrestricted use, distribution, and reproduction in any medium, provided the original author and source are credited.

\begin{abstract}
Large cohort epidemiological studies have shown a U-shaped association between the relative risk of Sudden Cardiac Death (SCD) and the dose of alcohol consumed across both genders. The mechanisms of this relationship, i.e., too little or too high promoting SCD while moderate doses providing protection, remain elusive. In this brief review, we provide plausible electrophysiological and biochemical mechanisms of the U-shaped association of the dose of alcohol and the risk of SCD. At moderate doses alcohol causes partial cellular uncoupling "paradoxically" improves the conduction at narrow ventricular conducting pathways (source) that adjoins a large tissue mass (sink) eliminating preexisting Unidirectional Conduction (UCB) (from source-to-sink) and preventing re-entry formation. In contrast, higher doses of alcohol by further increasing cellular uncoupling cause conduction block at multiple ventricular sites (wavebreaks) in isolated tissues and patterned monolayers of cardiac myocytes leading to the formation of multiple reentrant and non-reentrant wave fronts simulating fibrillation-like state. In the absence of alcohol, the vulnerable narrow conducting pathways remain unchanged promoting UCB (i.e., conduction block from source-to-sink block) increasing the risk of reentry formation. Re-entry leads to Ventricular Fibrillation (VF), a major cause of SCD. Our hypothesis of U-shaped association between alcohol dose and SCD is based on the preponderance of clinical epidemiological data and on basic experimental studies using intact animal hearts and patterned monolayers of cardiac myocytes with vulnerable narrow strands of conducting pathways (source) abutting to a large tissue mass (sink).
\end{abstract}

\section{Introduction}

It has long been recognized that acute alcohol intoxication can cause death from accidents or violence and that long-term misuse can increase the incidence of certain kinds of cancer and multi-organ diseases affecting the heart and the liver. According to a 2014 report prepared by the World Health Organization, Global Status Report on Alcohol and Health, an alcohol attributable death occurs every 10 seconds. Yet, increasing evidence suggests that regular consumption of light-to-moderate amounts of alcohol (i.e., one to one and one half standard units) can reduce the risk of Ischemic Heart Disease (IHD) and Sudden Cardiac Death (SCD) [1-6]. These same studies uniformly reported that higher doses of chronic alcohol consumption (>3 standard units) cancel the beneficial cardiac effects of the lower doses of alcohol, promoting Coronary Artery Disease (CAD), SCD, cardiomyopathy, hypertension, and heart failure $[7,8]$. Cardiac and multi-organ diseases induced by chronic higher doses ( $>3$ units) of alcohol consumption result in an average of 22 years of reduction in life expectancy compared to the general population [9]. According to the National Institute on Alcohol Abuse and Alcoholism, one U.S. "standard" drink contains $\sim 15$ grams of pure alcohol, which is found in 12 ounces of regular beer ( $5 \%$ per volume alcohol), in five ounces of wine ( $12 \%$ per volume alcohol) and 1.5 ounces of spirits $(\sim 40 \%$ alcohol). Therefore, up to $15 \mathrm{~g} /$ day is considered "light-to-moderate," and greater than $30 \mathrm{~g} /$ day is considered "high" [3,4].

The potential benefits of light-to-moderate doses of alcohol consumption, however, has not received universal acceptance. A recent systematic review of epidemiological data by The Global Burden of Disease Study by Alcohol Collaborators challenged the health benefits of light-to-moderate levels of alcohol consumption. This meta-analysis concluded that the level of alcohol consumption that minimized harm "across all health outcomes particularly the risk of cancer, was zero" [10]. This seeming controversy stems from the fact that certain diseases like Atrial Fibrillation (AF), stroke, [11] hypertension, heart failure, cardiomyopathy, cancer, and liver disease do not manifest any benefit from alcohol, [5,12,13] while other diseases do. The potential mechanisms of the observed benefits of low-to-moderate alcohol consumption in CAD and SCD remain undefined because the observed epidemiologic data are associative and not causative, providing no mechanistic insight by which alcohol causes it's beneficial or its ill effects. Since the questions that motivate 
most studies in the health sciences are not associational but causal in nature, it is important to elucidate the possible underlying biological processes involved for preventive and therapeutic purposes. Whenever possible, such knowledge can be acquired by clinical studies and through more aggressive investigations using animal models of human disease. Here, we provide potential mechanisms of CAD and SCD and discuss in some details the $\mathrm{U}$-shaped response of SCD to increasing doses of alcohol.

\section{Epidemiological studies on SCD and CAD}

In a landmark study in 1994, Doll and Associates determined the incidence of all-cause mortality in relation to the average amount of pure alcohol consumed per day. The study was performed on 12,000 male middle and older aged British doctors, and was followed-up over 13 years. Those who reported drinking an average of $20 \mathrm{~g} /$ day had significantly lower all-cause mortality than those who consumed no alcohol. However, when the consumption became greater than 30 $\mathrm{g} /$ day, all-cause mortality increased. In other words, too little or too much alcohol intake was associated with a higher risk of death than moderate intake of alcohol. These opposing effects of alcohol produced a U-shaped relationship between the relative risk of all-cause mortality and the amount of alcohol consumed [3]. The figure 1 illustrates schematically the U-shaped relationship between the relative risk of mortality and the dose of alcohol based on multiple epidemiological studies. This large cohort study confirmed an earlier observation made on a smaller cohort involving 152 subjects of both sexes. This study suggested that 1-3 standard drinks per day reduced the risk of death caused by sudden pulselessness [1]. The results of these earlier studies are consistent with recent meta-analysis studies demonstrating selective benefits of low-to-moderate alcohol consumption in reducing the relative risk of $\mathrm{CAD}$ that causes ischemic heart disease [14] and SCD [4]. Furthermore, a meta-analysis, conducted across both genders, that included more than 84 studies, demonstrated a selective benefit in drinkers of $<60 \mathrm{~g} /$ day alcohol compared to non-

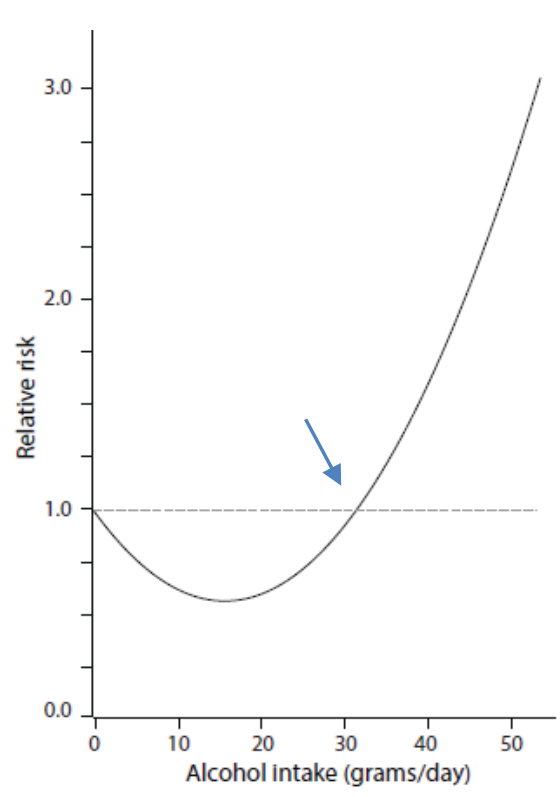

Figure 1: Schematic U-shaped curve representation of relative risk of sudden cardiac death relative to alcohol intake observed in large epidemiological studies. Notice that consumption of $>30 \mathrm{~g} /$ day (arrow) raises the relative risk as uniformly shown in multiple independent epidemiological studies in both genders. drinkers, [15] consistent with previous studies [1,3,14]. However, alcohol's cardioprotective effect on IHD mortality was lost when alcohol consumption was above $60 \mathrm{~g} /$ day [15].

The mechanisms by which low doses of alcohol reduce the risk of myocardial ischemia remain elusive. While the antioxidant effects of red wine (The French Paradox) [16] was proposed as a potential mechanism in preventing $\mathrm{CAD}$, it is becoming clear that it is actually the dose of pure alcohol and not the source (e.g., wine, beer, liquor) that provides risk reduction of cardiac disease [4]. However, not everyone agrees with this as "non-alcoholic components of alcoholic beverages" (i.e., polyphenols) may also play a role in the protective effects of alcoholic beverages on health. Multiple factors may contribute to alcohol's protective effect against CAD. The two major consequences of alcohol's modulation of these risk factors are: reduction in the risk of blood clot formation and prevention of intravascular plaque development and rupture. Alcohol's effects on these risk factors include, but are not necessarily limited to, the activation of the fibrinolytic system, lowering of platelet aggregation, and improved endothelial function [1,17]. Importantly, lowering LDL cholesterol (bad cholesterol) [18] and elevating HDL cholesterol (good cholesterol) while reducing C-reactive protein levels greatly diminish the risk of CAD $[18,19]$. It is thought that these effects of alcohol on blood constituents and improving the lipid profile reduce the risk of atherosclerosis by preventing plaque buildup in the coronary arteries and preventing the formation of blood clots, reducing the risk of myocardial ischemia $[6,19]$. Interestingly, a recent study found that red wine consumption increases coronary artery calcification which increases plaque stability, significantly reducing the risk of myocardial ischemia and infarction compared to non-drinkers [19]. Whether or not this effect is related to pure alcohol or the antioxidant constituents of red wine remains to be defined.

While epidemiological data on pooled dose-response data based on 28 cohort studies have shown an association between heavy alcohol consumption and increased risk of IHD, the causal mechanisms of such an association are complex and multifactorial [20]. No randomized controlled clinical trial has tried to establish and unravel the mechanism of increased risk of IHD caused by CAD in heavy smokers. Such trials would be extremely difficult to conduct given the almost obligatory presence of confounding risk factors such as smoking, hypertension, and misdiagnosis of cardiomyopathy as CAD [8]. It must be noted that prolonged chronic heavy drinking can result in the most extreme form of cardiac tissue damage, cardiomyopathy [21].

\section{Potential mechanism of U-shaper response of SCD}

Interestingly, patients specifically stratified by risk of SCD manifest a U-shaped relationship between the dose of alcohol and the risk of SCD. The mechanism of SCD, i.e., death occurring within one hour of symptom onset (unconsciousness) [4] is most often caused by Ventricular Fibrillation (VF), [22-26] a rapid, disorganized activation of the ventricles [27-30]. A potential confounding factor of alcoholmediated VF may result from the fact that alcohol also promotes AF, which in turn increases the risk of VF. Some $35 \%$ to $62 \%$ of instances of Paroxysmal AF (PAF) presented to an emergency room are alcoholrelated, with onset frequently 12 to 36 hours after cessation of binge drinking (Holiday Heart Syndrome) [11]. The NHLBI's Cardiovascular Health Study cohort study showed that the incidence rate of SCD is higher in patients with AF (2.9 per 1000 per year) compared with non-AF controls (1.3 per 1000 per year) [31]. Today an estimated 2.76.1 million people in the United States have AF, affecting more than 33.5 million people worldwide AF. With the aging of the U.S. and world population, this number is expected to increase [31]. Since the 
relationship between AF risk and the dose of alcohol is linear, [11] it seems clear that the recent observation of a $\mathrm{U}$-shaped relationship between the dose of alcohol and the risk of SCD must result from trigger mechanisms other than AF. A large prospective cohort study involving 85,067 women from the Nurses' Health Study (currently known as the Women's Health Study) evaluated the relationship between the dose of alcohol consumed and the risk of SCD in the absence of AF [4]. The study, which began in 1976 and continued until 2004, was conducted on women aged 30-55 years during entry into the study and who were free of chronic diseases. The SCD cases in this study were separated from the non-SCD cases and were documented by medical records and through reports from next of kin [4]. During this study period, SCD occurred in 295 cases, non-sudden coronary heart disease deaths in 987 cases, and nonfatal Myocardial Infarction (MI) in 2,195 cases [4]. The relative risk of alcohol in causing VF (SCD) in this study was found to be U-Shaped and was adjusted for other major risk factors known to directly or indirectly affect the relative risk of VF. These risk factors included coronary heart disease, hypertension, diabetes, body mass index, parental history of MI, smoking, age, and physical activity. Chiuve, et al. [4] identified a U-shaped association between alcohol intake and risk of SCD. Specifically, they fit a Cox proportional hazards model to estimate the relative risk of SCD for various levels of alcohol consumption and controlling for various covariates, like smoking, hypertension, diabetes [4]. To test for the $\mathrm{U}$-shaped relation, they fit a model of the form: $\lambda\left(\frac{t}{X, Y}\right)=\lambda_{0}(t) e^{\alpha Y}+\beta X^{2}$, where $X$ is a continuous measure of alcohol consumption and $X$ represent other covariates such as smoking, age, etc. The $X^{2}$ term is what lends the model its $U$ (curvilinear) shape. This model was found to be statistically significant (p-value of the coefficient term $\beta$ was $<0.02$ adjusted for different sets of covariates). Interestingly, when the analogous coefficient incorporated $X$ instead of $X^{2}$, the model exhibited a linear relationship and most importantly was not statistically significant. While this may suffer recall bias (the participants were asked to recall how often they consumed alcohol over the past), nevertheless given the relatively large number of participants the demonstration of U-shaped association remains valid [4]. Among women who consumed 5 to 15 $\mathrm{g}$ /day of alcohol, the relative risk of SCD was significantly reduced compared to abstainers. With this respect, the relative risk of SCD dropped by 36 percent when women consumed between one-half to one drink per day [4]. However, the relative risk of SCD increased when consumption exceeded $30 \mathrm{~g} /$ day. These results are consistent with earlier studies in males and in subjects of both genders [3,32]. Interestingly, alcohol's beneficial effect on SCD was independent of its source, e.g., beer $v s$ wine $v s$ spirits, [4] suggesting additional factors providing benefit other than the antioxidants present in red wine proposed in the French Paradox [16]. Presently, there is no study that provides insight into the mechanism(s) of alcohol's pro- and anti-VF effect. It then becomes clear that an understanding of the mechanisms of a U-shaped association between the level of alcohol and the relative risk of SCD is based on defining the mechanism(s) by which alcohol exerts its anti-VF effect at low doses but pro-VF effect at high doses.

\section{Potential mechanisms of VF}

$\mathrm{VF}$ is characterized by multiple wavelets and constantly forming foci caused by triggered activity $[23,33,34]$. Here, we propose possible electrophysiological and biochemical signaling mechanisms of alcohol's dose-dependent pro-VF and anti-VF effect. Microscopic and macroscopic cardiac tissue structural discontinuities profoundly modulate cardiac depolarizing wave conduction, promoting Unidirectional Conduction Block (UCB), re-entrant excitation, and increased risk of VF [35-37]. Multiple factors cause tissue discontinuities, like muscular trabeculae (small muscular bridges connecting large tissue mass) and increased aging and disease-induced cardiac interstitial fibrosis [30,38,39]. Discontinuous propagation and UCB occur when propagation proceeds through a narrow strand and suddenly encounters a large mass of tissue. Under these conditions, a mismatch between the upstream depolarizing current "source" in the narrow strand and the downstream repolarizing current "sink" (i.e., large mass of tissue at rest) develops preventing the wave to propagate from the narrow strands to a larger tissue bulk. However, impulses can propagate from the larger tissue mass (large source) to the narrow strands (small sink) as demonstrated in patterned monolayers of cardiac myocytes, [40-43] and in isolated cardiac tissues [35-37] and in explanted human hearts with cardiomyopathy [44]. Electrical propagation at the very site of source-to-sink mismatch (i.e., the site of sudden tissue expansion) is greatly affected by the degree of local cell-to-cell coupling. Partial uncoupling only minimally affects the upstream source in the narrow strands of muscles but greatly diminishes the downstream sink effect caused by increased tissue impedance [43]. This asymmetrical effect is termed "paradoxical" because of the greater influence of partial cellular uncoupling on the sink rather than the source, an effect that suppresses UCB and restores bidirectional conduction patterned tissue culture $[40,43]$ and in simulation studies [45]. Further increases in cellular uncoupling promote bidirectional conduction block, wavebreaks, and formation of multiple wavelets [28]. These findings indicate partial uncoupling restores normal conduction at sites with UCB while further increases in degrees of uncoupling promote bidirectional conduction block and wavebreak $[40,43]$. It then follows that drug-induced partial cellular uncoupling imparts non-symmetrical effects on the direction of impulse propagation in discontinuous cardiac tissue [43]. Aliphatic alcohols such as heptanol, [46] ethanol, [47] and palmitoleic acid [40] cause partial cellular uncoupling at low doses and complete uncoupling at higher doses, with ethanol being the least potent cellular uncoupler [47]. Low doses of aliphatic alcohols slightly slow conduction velocity due to partial uncoupling without affecting the source, i.e., fast $\mathrm{Na}$ current both in atria [46] and in ventricular tissue [48]. Wavebreaks with higher doses of ethanol promote the formation of multiple wavelets [47] simulating fibrillation-like state [49]. These experimental findings raise the hypothesis that low concentrations of alcohol, by inducing partial cellular uncoupling, eliminates UCB and protects the heart against reentrant VT/VF, while higher doses cause conduction block (wavebreaks) leading to VF. Higher doses may of alcohol also produce VF by prolonging the QT interval in humans increasing the dispersion of repolarization and the risk of torsade-depoints (VT) and VF [50,51]. Electrophysiological studies in humans have shown that intravenous alcohol administration in heavy chronic drinkers promotes electrically inducible VT/VF [51]. It is highly likely that the interaction between dispersion of repolarization and discontinuous propagation act synergistically to promote reentry and VF at macroscopic size scale [41]. Higher doses of ethanol and its metabolite the acetaldehyde reduce inward rectifier potassium current $\left(\mathrm{IK}_{1}\right)[52,53]$. Reduced repolarization facilitates the emergence of after potentials [45] and the risk of VT/VF. In addition, higher doses of chronic administration of alcohol in mice alter the distribution of gap junctional Ca43 proteins and increase the structural discontinuities of ventricular tissue. Under these conditions, the vulnerability to VF increases as evidenced by the increased susceptibility to electrically inducible VT/VF in these mice [54].

\section{Potential molecular pathways of VF}

Preliminary evidence suggests that the neurophysiological response 
to low-to-moderate doses of alcohol [55] may reduce the risk of VF by reducing the levels of stress and anxiety through decreasing the sympathetic outflow to the heart. Increased sympathetic outflow to the heart increases the risk of SCD [56-58] and IHD [6,59]. The improvement of overall feelings and performance with low dose alcohol was suggested be mediated, at least in part, by the calming and/or sedative effects of the alcohol [60]. However, not all studies subscribed to this scenario. For example, in alcoholics, alcohol ingestion does not appear in general to relieve anxiety and may increase anxiety in susceptible subjects during a drinking binge. Clearly, more research is needed to assess the validity of the anxiety-reducing theory for alcohol abuse [6]. These findings suggest that low dose alcohol as a stress reducing agent requires individualized assessment of each subject by the caring physician as it cannot be prescribed as a "drug" to reduce tension across different individuals [61]. Advice to consume any amount of alcohol must balance the potential benefits against potential risks related to other chronic diseases.

Finally, in addition to our hypothesized electrophysiological mechanisms, activation of the stress enzyme c-Jun N-Terminal Kinase (JNK) [62] phosphorylates and activates the arrhythmogenic cardiac Ca-calmodulin-dependent protein kinase II (CaMKII) ("kinase on kinase") [63]. Activation of the highly arrhythmogenic CaMKII [64] increases the late inward $\mathrm{Na}$ and $\mathrm{Ca}$ currents, promoting afterpotentials and rapid triggered activity [65] leading to VT/ VF $[30,66,67]$ and AF $[67,68]$. By increasing cardiac sympathetic activity, high doses of alcohol increase the "leakiness" of $\mathrm{Ca}^{2+}$ from the Sarcoplasmic Reticulum (SR) via the ryanodine receptors promoting calcium waves, early and delayed afterdepolarizations (EAD \& DADs respectively) that initiate rapid triggered activity leading to AF and VF [68-72].

\section{Conclusions}

This mini-review is based on a large number of prior epidemiological studies showing light-to-moderate doses of alcohol (ethanol) consumption reduce the risk of SCD while abstinence or higher doses increase the risk of SCD caused by VF. Based on alcohol's ability to cause dose-dependent cellular electrical uncoupling, we postulated that lowto-moderate doses of alcohol prevent Unidirectional Block (UCB), reentry and VF while very little or no alcohol consumption preserve UCB and the vulnerability to reentry and VF. In contrast, higher doses of alcohol increases cellular uncoupling promoting conduction block, reentry and VF. Multiple additional mechanisms are also involved in promoting VF with toxic doses of alcohol including activation of the arrhythmogenic enzyme CaMKII. Recommendation of alcohol use to reduce the risk of SCD must however, be strictly individualized by the caring physician and not be contraindicated by other factors including atrial fibrillation. Here, it seems pertinent to conclude with a humorous quote from the founder of evolutionary biology, Charles Darwin, who stated, "An American monkey, after getting drunk on brandy, would never touch it again, and thus is much wiser than most men."

\section{References}

1. Siscovick DS, Weiss NS, Fox N (1986) Moderate Alcohol Consumption and Primary Cardiac Arrest. Am J Epidemiol 123: 499-503.

2. Marmot M, Brunner E (1991) Alcohol and Cardiovascular Disease: The Status of the U Shaped Curve. BMJ 303: 565-568.

3. Doll R, Peto R, Hall E, Wheatley K, Gray R (1994) Mortality in Relation to Consumption of Alcohol: 13 Years' Observations on Male British Doctors. BMJ 309: 911-918.
4. Chiuve SE, Rimm EB, Mukamal KJ, Rexrode KM, Stampfer MJ, et al. (2010) Light-to-Moderate Alcohol Consumption and Risk of Sudden Cardiac Death in Women. Heart Rhythm 7: 1374-1380.

5. Fernandez-Sola J (2015) Cardiovascular Risks and Benefits of Moderate and Heavy Alcohol Consumption. Nat Rev Cardiol 12: 576-587.

6. Piano MR (2017) Alcohol's Effects on the Cardiovascular System. Alcohol Res 38: 219-241.

7. Dguzeh U, Haddad NC, Smith KTS, Johnson JO, Doye AA, et al. (2018) Alcoholism: A Multi-Systemic Cellular Insult to Organs. Int J Environ Res Public Health 15: 1083.

8. Klatsky AL (2015) Alcohol and Cardiovascular Diseases: Where Do We Stand Today? J Intern Med 278: 238-250.

9. Rehm J, Guiraud J, Poulnais R, Shield KD (2018) Alcohol Dependence and Very High Risk Level of Alcohol Consumption: A Life-Threatening and Debilitating Disease. Addict Biol 23: 961-968.

10. Collaborators GBDA (2018) Alcohol Use and Burden for 195 Countries and Territories, 1990-2016: A Systematic Analysis for the Global Burden of Disease Study. Lancet 392: 1015-1035.

11. Voskoboinik A, Prabhu S, Ling LH, Kalman JM, Kistler PM (2016) Alcohol and Atrial Fibrillation: A Sobering Review. J Am Coll Cardiol 68: 2567-2576.

12. Seitz HK, Bataller R, Cortez-Pinto H, Gao B, Gual A, et al. (2018) Alcoholic Liver Disease. Nat Rev Dis Primers 4: 16.

13. Kypri K, McCambridge J (2018) Alcohol Must be Recognised as a Drug. BMJ 362: k3944.

14. Roerecke M, Rehm J (2014) Alcohol Consumption, Drinking Patterns, and Ischemic Heart Disease: A Narrative Review of Meta-Analyses and a Systematic Review and Meta-Analysis of the Impact of Heavy Drinking Occasions on Risk for Moderate Drinkers. BMC Med 12: 182.

15. Ronksley PE, Brien SE, Turner BJ, Mukamal KJ, Ghali WA (2011) Association of Alcohol Consumption With Selected Cardiovascular Disease Outcomes: A Systematic Review and Meta-Analysis. BMJ 342: d671.

16. Providencia R (2006) Cardiovascular Protection From Alcoholic Drinks: Scientific Basis of the French Paradox. Rev Port Cardiol 25: 1043-1058.

17. Hennekens CH, Rosner B, Cole DS (1978) Daily Alcohol Consumption and Fatal Coronary Heart Disease. Am J Epidemiol 107: 196-200.

18. Vu KN, Ballantyne CM, Hoogeveen RC, Nambi V, Volcik KA, et al. (2016) Causal Role of Alcohol Consumption in an Improved Lipid Profile: The Atherosclerosis Risk in Communities (ARIC) Study. PLoS One 11: e0148765.

19. da Luz PL, Favarato D, Moriguchi EH, de Carli W, Bruscato N, et al. (2018) Red Wine Consumption, Coronary Calcification, and LongTerm Clinical Evolution. Braz J Med Biol Res 51: e7703.

20. Corrao G, Rubbiati L, Bagnardi V, Zambon A, Poikolainen K (2000) Alcohol and Coronary Heart Disease: A Meta-Analysis. Addiction 95: 1505-1523.

21. Maisch B (2016) Alcoholic Cardiomyopathy: The Result of Dosage and Individual Predisposition. Herz 41: 484-493.

22. NHLBI (2016) What Causes Sudden Cardiac Arrest? National Institute of Health, USA. 
23. Weiss JN, Karma A, Shiferaw Y, Chen PS, Garfinkel A, et al. (2006) From Pulsus to Pulseless: The Saga of Cardiac Alternans. Circ Res 98 1244-1253.

24. Zipes DP, Wellens HJJ (1998) Sudden Cardiac Death. Circulation 98: 2334-2351.

25. Myerburg RJ, Kessler KM, Castellanos A (1993) Sudden Cardiac Death: Epidemiology, Transient Risk, and Intervention Assessment. Ann Intern Med 119: 1187-1197.

26. Goldberger JJ, Basu A, Boineau R, Buxton AE, Cain ME, et al. (2014) Risk Stratification for Sudden Cardiac Death: A Plan for the Future. Circulation 129: 516-526.

27. Karagueuzian HS (2016) Synergism between Enhanced Late Inward Currents and Tissue Fibrosis in the Initiation of Spontaneous Ventricular Tachyarrhythmias. J Heart Health 2.

28. Weiss JN, Qu Z, Chen PS, Lin SF, Karagueuzian HS, et al. (2005) The Dynamics of Cardiac Fibrillation. Circulation 112: 1232-1240.

29. Weiss JN, Garfinkel A, Karagueuzian HS, Nguyen TP, Olcese R, et al. (2015) Perspective: A Dynamics-Based Classification of Ventricular Arrhythmias. J Mol Cell Cardiol 82: 136-152.

30. Morita N, Sovari AA, Xie Y, Fishbein MC, Mandel WJ, et al. (2009) Increased Susceptibility of Aged Hearts to Ventricular Fibrillation During Oxidative Stress. Am J Physiol Heart Circ Physiol 297: H1594-H1605.

31. Rattanawong $\mathrm{P}$, Upala $\mathrm{S}$, Riangwiwat $\mathrm{T}$, Jaruvongvanich $\mathrm{V}$, Sanguankeo A, et al. (2018) Atrial Fibrillation is Associated with Sudden Cardiac Death: A Systematic Review and Meta-Analysis. J Interv Card Electrophysiol 51: 91-104.

32. Wannamethee G, Shaper AG (1992) Alcohol and Sudden Cardiac Death. Br Heart J 68: 443-448.

33. Sato D, Xie LH, Sovari AA, Tran DX, Morita N, et al. (2009) Synchronization of Chaotic Early Afterdepolarizations in the Genesis of Cardiac Arrhythmias. Proc Natl Acad Sci USA 106: 2983-2988.

34. Morita N, Lee JH, Xie Y, Sovari A, Qu Z, et al. (2011) Suppression of Re-Entrant and Multifocal Ventricular Fibrillation by the Late Sodium Current Blocker Ranolazine. J Am Coll Cardiol 57: 366-375.

35. Wit AL, Hoffman BF, Cranefield PF (1971) Slow Conduction, Reentry, and the Mechanism of Ventricular Arrhythmias in Myocardial Infarction. Bull N Y Acad Med 47: 1233-1234.

36. Wit AL, Hoffman BF, Cranefield PF (1972) Slow Conduction and Reentry in the Ventricular Conducting System. Circ Res 30: 1-10.

37. Gotoh M, Uchida T, Mandel WJ, Fishbein MC, Chen PS, et al. (1997) Cellular Graded Responses and Ventricular Vulnerability to Reentry by a Premature Stimulus in Isolated Canine Ventricle. Circulation 95 : 2141-2154.

38. Ono N, Hayashi H, Kawase A, Lin SF, Li H, et al. (2007) Spontaneous Atrial Fibrillation Initiated by Triggered Activity Near the Pulmonary Veins in Aged Rats Subjected to Glycolytic Inhibition. Am J Physiol Heart Circ Physiol 292: 639-648.

39. Hayashi H, Wang C, Miyauchi Y, Omichi C, Pak HN, et al. (2002) AgingRelated Increase to Inducible Atrial Fibrillation in the Rat Model. J Cardiovasc Electrophysiol 13: 801-808.

40. Rohr S, Kucera JP, Fast VG, Kleber AG (1997) Paradoxical Improvement of Impulse Conduction in Cardiac Tissue by Partial Cellular Uncoupling. Science 275: 841-844.

41. Spach MS, Dolber PC, Heidlage JF (1989) Interaction of Inhomogeneities of Repolarization with Anisotropic Propagation in Dog Atria. A Mechanism for both Preventing and Initiating Reentry. Circ Res 65: 1612-1631.
42. Spach MS, Miller WT III, Geselowitz DB, Barr RC, Kootsey JM, et al. (1981) The Discontinuous Nature of Propagation in Normal Canine Cardiac Muscle. Evidence for Recurrent Discontinuities of Intracellular Resistance that Affect the Membrane Currents. Circ Res 48: 39-54.

43. Kucera JP, Rohr S, Kleber AG (2017) Microstructure, Cell-to-Cell Coupling, and lon Currents as Determinants of Electrical Propagation and Arrhythmogenesis. Circ Arrhythm Electrophysiol 10: e004665.

44. Wu TJ, Ong JJ, Hwang C, Lee JJ, Fishbein MC, et al. (1998) Characteristics of Wave Fronts During Ventricular Fibrillation in Human Hearts with Dilated Cardiomyopathy: Role of Increased Fibrosis in the Generation of Reentry. J Am Coll Cardiol 32: 187-196.

45. Xie Y, Sato D, Garfinkel A, Qu Z, Weiss JN (2010) So Little Source, So Much Sink: Requirements for Afterdepolarizations to Propagate in Tissue. Biophys J 99: 1408-1415.

46. Ohara T, Qu Z, Lee MH, Ohara K, Omichi C, et al. (2002) Increased Vulnerability to Inducible Atrial Fibrillation Caused by Partial Cellular Uncoupling with Heptanol. Am J Physiol Heart Circ Physiol 283: H1116-H1122.

47. Podgurskaya AD, Tsvelaya VA, Frolova SR, Kalita IY, Kudryashova NN, et al. (2018) Effect of Heptanol and Ethanol on Excitation Wave Propagation in a Neonatal Rat Ventricular Myocyte Monolayer. Toxicol In Vitro 51: 136-144.

48. Jalife J, Sicouri S, Delmar M, Michaels DC (1989) Electrical Uncoupling and Impulse Propagation in Isolated Sheep Purkinje Fibers. Am J Physiol 257: H179-H189.

49. Garfinkel A, Chen PS, Walter DO, Karagueuzian HS, Kogan B, et al. (1997) Quasiperiodicity and Chaos in Cardiac Fibrillation. J Clin Invest 99: 305-314.

50. Rossinen J, Sinisalo J, Partanen J, Nieminen MS, Viitasalo M (1999) Effects of Acute Alcohol Infusion on Duration and Dispersion of QT Interval in Male Patients with Coronary Artery Disease and in Healthy Controls. Clin Cardiol 22: 591-594.

51. Kupari M, Koskinen P (1998) Alcohol, Cardiac Arrhythmias and Sudden Death. Novartis Found Symp 216: 68-79.

52. Yamakura T, Lewohl JM, Harris RA (2001) Differential Effects of General Anesthetics on G Protein-Coupled Inwardly Rectifying and Other Potassium Channels. Anesthesiology 95: 144-153.

53. Horakova Z, Matejovic P, Pasek M, Hosek J, Simurdova M, et al. (2016) Effect of Ethanol and Acetaldehyde at Clinically Relevant Concentrations on Atrial Inward Rectifier Potassium Current IK1: Separate and Combined Effect. J Physiol Pharmacol 67: 339-351.

54. Lai YJ, Hung CL, Hong RC, Tseng YM, Lin Cl, et al. (2011) Slow Conduction and Gap Junction Remodeling in Murine Ventricle After Chronic Alcohol Ingestion. J Biomed Sci 18: 72.

55. Gruenewald PJ, Mair C (2015) Heterogeneous Dose-Response and College Student Drinking: Examining Problem Risks Related to Low Drinking Levels. Addiction 110: 945-954.

56. Chen PS, Chen LS, Cao JM, Sharifi B, Karagueuzian HS, et al. (2001) Sympathetic Nerve Sprouting, Electrical Remodeling and the Mechanisms of Sudden Cardiac Death. Cardiovasc Res 50: 409-416.

57. Shen MJ, Zipes DP (2014) Role of the Autonomic Nervous System in Modulating Cardiac Arrhythmias. Circ Res 114: 1004-1021.

58. Ajijola OA, Lux RL, Khahera A, Kwon O, Aliotta E, et al. (2017) Sympathetic Modulation of Electrical Activation in Normal and Infarcted Myocardium: Implications for Arrhythmogenesis. Am J Physiol Heart Circ Physiol 312: H608-H621. 
59. Toschi-Dias E, Rondon MUPB, Cogliati C, Paolocci N, Tobaldini E, et al. (2017) Contribution of Autonomic Reflexes to the Hyperadrenergic State in Heart Failure. Front Neurosci 11: 162.

60. Lloyd HM, Rogers PJ (1997) Mood and Cognitive Performance Improved by a Small Amount of Alcohol Given with a Lunchtime Meal. Behav Pharmacol 8: 188-195.

61. Warren GH, Raynes AE (1972) Mood Changes During Three Conditions of Alcohol Intake. Q J Stud Alcohol 33: 979-989.

62. Davis RJ (2000) Signal Transduction by the JNK Group of MAP Kinases. Cell 103: 239-252.

63. Yan J, Thomson JK, Zhao W, Gao X, Huang F, et al. (2018) Role of Stress Kinase JNK in Binge Alcohol-Evoked Atrial Arrhythmia. J Am Coll Cardiol 71: 1459-1470.

64. Anderson ME (2007) Multiple Downstream Proarrhythmic Targets for Calmodulin Kinase II: Moving Beyond an Ion Channel-Centric Focus. Cardiovasc Res 73: 657-666.

65. Xie LH, Chen F, Karagueuzian HS, Weiss JN (2009) Oxidative StressInduced Afterdepolarizations and Calmodulin Kinase II Signaling. Circ Res 104: 79-86.

66. Bapat A, Nguyen TP, Lee JH, Sovari AA, Fishbein MC, et al. (2012) Enhanced Sensitivity of Aged Fibrotic Hearts to Angiotensin II\& Hypokalemia-Induced Early Afterdepolarizations-Mediated
Ventricular Arrhythmias. Am J Physiol Heart Circ Physiol 302: H2331-H2340.

67. Karagueuzian HS, Pezhouman A, Angelini M, Olcese R (2017) Enhanced Late $\mathrm{Na}$ and $\mathrm{Ca}$ Currents as Effective Antiarrhythmic Drug Targets. Front Pharmacol 8: 36.

68. Pezhouman A, Cao H, Fishbein MC, Belardinelli L, Weiss JN, et al. (2018) Atrial Fibrillation Initiated by Early AfterdepolarizationMediated Triggered Activity during Acute Oxidative Stress: Efficacy of Late Sodium Current Blockade. J Heart Health 4.

69. Ai X, Curran JW, Shannon TR, Bers DM, Pogwizd SM (2005) $\mathrm{Ca}^{2+} /$ Calmodulin-Dependent Protein Kinase Modulates Cardiac Ryanodine Receptor Phosphorylation and Sarcoplasmic Reticulum $\mathrm{Ca}^{2+}$ Leak in Heart Failure. Circ Res 97: 1314-1322.

70. Chelu MG, Sarma S, Sood S, Wang S, van Oort RJ, et al. (2009) Calmodulin Kinase II-Mediated Sarcoplasmic Reticulum $\mathrm{Ca}^{2+}$ Leak Promotes Atrial Fibrillation in Mice. J Clin Invest 119: 1940-1951.

71. Wit AL, Boyden PA (2007) Triggered Activity and Atrial Fibrillation. Heart Rhythm 4: S17-S23.

72. Priori SG, Chen SR (2011) Inherited Dysfunction of Sarcoplasmic Reticulum $\mathrm{Ca}^{2+}$ Handling and Arrhythmogenesis. Circ Res 108: 871 883 